\title{
Anomalous Light Propagation, Finite Size-Effects and Losses in Real 3D Photonic Nanostructures
}

\author{
Muriel Botey ${ }^{1}$, Gabriel Lozano ${ }^{2}$, Hernán Míguez ${ }^{2}$, Luís A. Dorado ${ }^{3}$, Ricardo A. Depine ${ }^{3}$, Jordi Martorel ${ }^{4,1}$ \\ ${ }^{1}$ Universitat Politècnica de Catalunya, EUETIB, Barcelona, Spain, e-mail: muriel.botey@upc.edu \\ ${ }^{2}$ Instituto de Ciencia de Materiales de Sevilla, CSIC, Sevilla, e-mail: hernan@cartuja.csic.es \\ ${ }^{3}$ Universidad de Buenos Aires, Buenos Aires, Argentina, e-mail: rdep@df.uba.ar \\ ${ }^{4}$ ICFO-Institut de Ciències Fotòniques, Castelldefels (Barcelona), Spain, e-mail: jordi.martorell@icfo.es
}

\begin{abstract}
The propagation of light through 3D ordered photonic nanostructures is strongly affected by real aspects of actual crystals, especially at the spectral range where the wavelength of light is on the order of the lattice parameter. We perform a theoretical study on anomalous light propagation in finite thin artificial opal slabs made of a reduced number of layers. The vector KKR method we use accounts for the finite character of the structure and for different sources of losses. We show that an interplay between finite-size effects and losses engineers group velocity at this high energy range. Only depending on the crystal size and extinction, light propagation can either be superluminal (positive or negative) or approach zero for certain frequencies. The numerically calculated group index is in good agreement with experimental results some of which remained partially unexplained.
\end{abstract}

Keywords: Photonic bandgap materials; nanomaterials; finite-size effects; slow light; fast light.

\section{INTRODUCTION}

Materials that can either provide a reduction of the speed of light or superluminal light propagation have recently attracted significant interest [1],[2]. Among the variety of systems that hold anomalous light propagation are two and three-dimensional (3D) periodically nanostructured materials [3]. In such systems, while low dispersive modes are associated with strong group velocity reductions [3], experiments have opal films, either in the $\Gamma L$ and $\Gamma X$ directions [4]-[7]. Several theoretical works have appeared to explain these new optical features [6],[7] and also interesting applications have been proposed to take advantage of such special optical properties at the high energy range of periodic nanostructures [7],[9].

In the present paper, we perform a comprehensive analysis on the influence of finite-size effects on the group index of thin artificial opals, in a wide energy spectral range and different propagation directions. For a perfect infinite system the geometry of the structure gives rise to extremely flat bands for $1.05 \leq a / \lambda \leq 1.35$ in the $\Gamma \mathrm{L}$ crystallographic direction. Also in the $\Gamma X$ direction, the slope of the photonic bands appears to be reduced at $\mathrm{X}$ high symmetry point. Such predictions coincide with experimental evidences of not only slow light but also fast light, in high quality opals with controlled thickness [4],[5]. In order to explain such observations, we adopt the same code used in refs. [7],[6] to model finite-width crystal slabs, taking into account the presence of a substrate. In the model, the effect of diffuse scattering due to structural imperfections is also accounted [6]. We accurately reproduce the observed finite-size effects on group velocity in both the $\Gamma \mathrm{X}$ and $\Gamma \mathrm{L}$ directions and show that they may also be strongly affected by the presence of losses.

\section{GROUP VELOCITY DETERMINATION}

Calculations are preformed using the KKR method, which is based on the code reported by Stefanou et al. [11],[12] developed from the vector Korringa-Kohn-Rostoker (KKR) method [13],[14]. This approach provides very accurate predictions for finite opals, requires reduced computational effort and faces the main important experimental aspects of actual crystals [7],[6]. For all calculations shown in this paper we consider facecentered-cubic (fcc) close-packed structures made of dielectric spheres, and take the same code parameters than in ref. [7]. From the condition of conservation of the tangential component of the wave vectors in a 2D closepacked triangular geometry, we can determine that, in the $\Gamma \mathrm{L}$ direction, diffraction in air starts at $a / \lambda \sim 1.63$ $(\sim 1.07$ in glass). Thus, upon normal incidence on the [111] planes, and for $a / \lambda \leq 1.63$, the only reflected nonvanishing mode is the specular one. For the case of transmission, for $a / \lambda \leq 1.4$, the forwardly transmitted, field dominates transmittance even for a glass supported opal. Also in the $\Gamma X$ direction for $a / \lambda \leq 1.35$, the only reflected non-vanishing mode is the specular one and also the forwardly transmitted mode dominates transmittance. Hence, despite the high complexity of the scattering phenomena that take place inside ordered nanostructures, for $1.0 \leq a / \lambda \leq 1.4$, the group velocity either in the $\Gamma \mathrm{X}$ or $\Gamma \mathrm{L}$ directions can be calculated from the fields that propagate normal to the [111] and [100] planes respectively [7],[6]. For propagation in a given crystallographic direction group velocity $\left(v_{g}\right)$ can be obtained from the phase delay, $\theta$, introduced by the structure to such forwardly transmitted field upon normal incidence on a given plain ensemble: $v_{g}=L(\partial \theta / \partial \omega)^{-1}$, where $L$ is the crystal thickness, $c$ the speed of light in vacuum. 


\section{FINITE SIZE EFFECTS ON THE GROUP VELOCITY}

At the high energy range the delay introduced to the forwardly transmitted electric field fluctuates depending on the sample thickness, indicating a strong influence of the presence of boundaries, see Fig. 1a. In the $\Gamma L$ direction, such anomalous dispersion gives rise to an anomalous $v_{g}$ at three particular frequency regions, namely $a / \lambda \sim 1.15, a / \lambda \sim 1.25$ and $a / \lambda \sim 1.30$. Since these spectral positions slightly depend on the actual crystal properties we refer to them as 'A', 'B', and 'C', respectively, as shown in Fig.1b. For the opal considered in Fig. 2b, a 6-[111]-layers-thick structure exhibits a highly negative group index value at the spectral position $\mathrm{A}$ and a positive small one at B; both corresponding to fast-light. Nevertheless, increasing the thickness to 10 layers, group index becomes negative at $\mathrm{B}$, which still corresponds to fast light, while we find slow light at $\mathrm{A}$. For a 20-[111]-layers-thick sample, the group velocity character changes: we recover fast light at $\mathrm{A}$, but with negative group index, while negative fast light at B becomes positive fast light. Therefore, for a given thickness, adding only one layer introduces a flip of the phase slope. Such phase jump translates into a change of the group index sign, from a negative group index to a large positive value. In Fig. 2 the slope flip at A takes place between 8 and 9 [111] layers, and at B between 14 and 15. Contrary to what could be expected, increasing the opal thickness does not cause the disappearance of the oscillations. A second flip takes place at A between 21 and 22-[111]-layers and even a third change in the $v_{g}$ sign is observed at this spectral position. Also at $\mathrm{B}, v_{g}$ has a second sign flip between 29 and 30 [111] planes. Finally, for already thick systems, further increasing the number of planes tends to smooth the fluctuations in this high-energy spectral range, either at $\mathrm{A}$ or $\mathrm{B}$. Nevertheless, rather than giving rise to a broad fast light regime, the average group velocity reduction is small, except for very narrow resonances. In the case of the frequency range denoted by $\mathrm{C}$, and for the extinction here considered, group index is negative for thin opal films and a positive superluminal regime persists even for thick structures.


Figure 1. a) Phase delay divided by the number of [111] planes, in $\pi$ units, introduced to the forwardly transmitted field propagating in the $\Pi$ direction, $\varepsilon_{\text {sph }}=2.5+0.06 i$. b) Corresponding group indexes, $n_{g}=c / v_{g}$ for different opal thickness. The figures inset indicate the number of [111] planes. All curves are plotted as a function of reduced frequency.

In the transitions between negative superluminal light propagation to a low positive $v_{g}$, the difference in the phase delay introduced to the forwardly transmitted electric field, adding one single layer, is exactly of $2 \pi$, see Fig. 2b. Such anomalous phase delay behavior was experimentally measured by Galisteo-López et al. at the frequency range A for a glass supported opal slab made of polystyrene spheres in air [5]. In that case, it was observed that the transition took place when the thickness was increased from 7 to 8 [111] layers. We reproduce such observations using KKR method with extinction, with a sphere's dielectric function taken in accordance to polystyrene dispersion [10] and consider a slight lattice relaxation. As shown in Fig.2a, taking $\varepsilon_{g}=2.34$ for the glass substrate and no other adjusting parameter, the calculations give the expected results. At $a / \lambda \sim 1.2$, for a thickness ranging from 4 to 7 layers the phase slope and hence the group index is negative. Our predictions confirm theoretically that for an 8-[111]-layers-thick opal, group index inverts its sign from negative to positive, changing from superluminal to slow light propagation, see Fig. 2a inset. Our numerical results, for different opal thicknesses (keeping all other parameters constant) confirm the $2 \pi$ phase shift at the exact frequency where the flip of the group index sign takes place, in excellent agreement with the results reported in ref. [5]. 

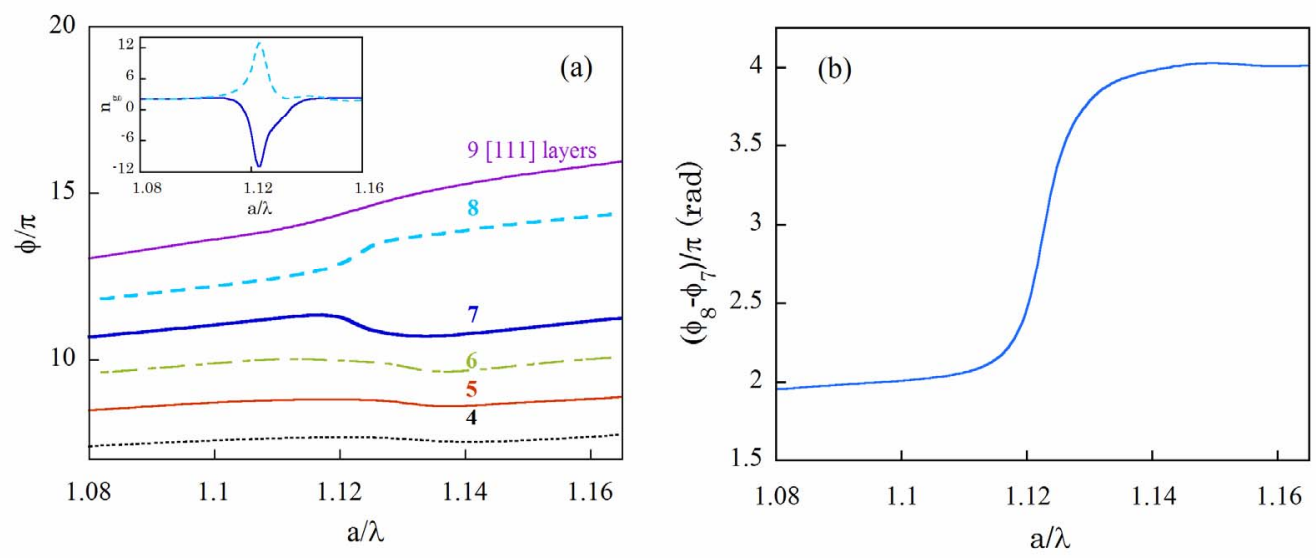

Figure 2. a) Calculated phase delay of the forwardly transmitted field in the A region, for different opal thickness indicated on the graph as the number of stacked [111] layers. Dielectric constant of the spheres and the glass support: $\varepsilon_{\text {shp }}=2.6+0.0575 i, \varepsilon_{g}=2.34$ [7]. b) Difference phase delay introduced to the transmitted field between 8 and 7 [111] layers, in $\pi$ units. All curves are plotted as a function of reduced frequency.

In the $\Gamma \mathrm{X}$ crystallographic direction, the dispersion relation shows abrupt changes in slope which translate into an anomalous $v_{g}$ for $a / \lambda \geq 0.9$. The group index displayed in Fig. 3 explains the experimental observations of ref. [4] where two abrupt changes in the measured phase slope give two group index peaks, at $a / \lambda \sim 1$ and $a / \lambda \sim 1.35$. We have reproduced these experimental observations taking into account the presence of a glass substrate in the calculations. Notice however that an unsupported opal provides almost the same results, see Fig. 3. Hence, we believe that the origin of such phase fluctuations and the group index peaks is to be found in the collective electromagnetic resonances within the ordered array, which are affected by the presence of imperfections and finite-size effects.

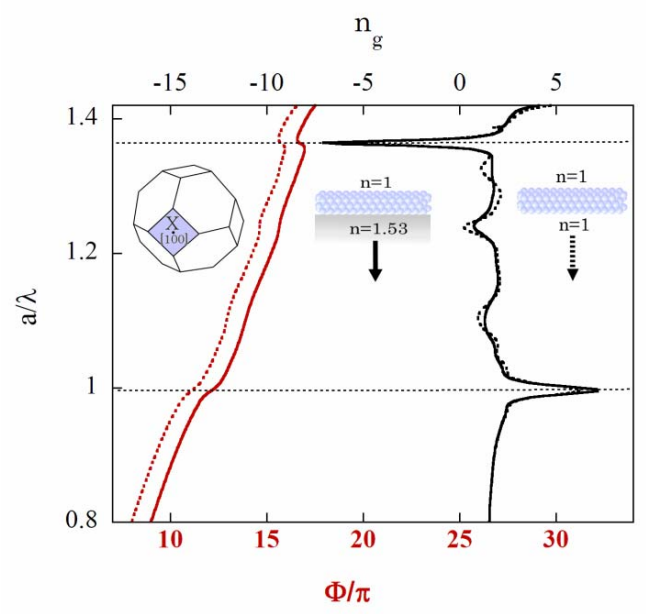

Figure 3. The red curves, shifted for clarity, display the phase delay, in $\pi$ units, introduced to the transmitted field in the $\Gamma X$ direction of a 7-[100]-layer glass supported -solid-or unsupported opal —dotted-. The black curves show the corresponding group index for the glass supported -solid-and unsupported opal —dotted-, $\varepsilon_{\text {shp }}=2.6+0.12 i$ and $\varepsilon_{g}=2.34$, respectively. The vertical axis displays the reduced frequency.

\section{THE ROLE OF EXTINCTION}

Disorder is present in any practical realization of periodic nanostructures and its effects are unavoidable. Moreover, while disorder has little effect on the optical spectra in the low-energy range, it dramatically affects the response of the system at the high-energy range. Hence, for high frequencies, $v_{g}$ does not only depend on the crystal geometry and dielectric contrast, but also the presence of imperfections plays a crucial role. For simplicity reasons, we do not distinguish between different sources of disorder and losses; we model extinction only by the introduction of an imaginary part to the sphere's dielectric function which accounts for the diffuse light scattering produced by imperfections, vacancies or dislocations, instead of managing through the use of more than one parameter [10],[15]. This method has already been successfully applied to colloidal crystals and 
validated with experimental data available in the literature [7],[6],[16],[15]. The values normally required for the imaginary part of the dielectric constant to fit the reflectance spectra of real samples range from $0.04 \mathrm{i}$ to $0.14 \mathrm{i}$ [6]. In the $\Gamma L$ direction, the group index transitions from negative to positive values depend on extinction. Fig. 4 shows how, for a given extinction the $v_{g}$ dependence on the opal thickness changes. In fact, increasing or decreasing such imaginary part of the dielectric function causes $v_{g}$, at a certain frequency, to change its sign. For instance, for a 10-[111]-layers-thick opal group index is either positive or negative for $0.06 \mathrm{i}$ or $0.1 \mathrm{i}$, respectively. For higher extinctions, the group index fluctuations smooth out in general at B and C. At these spectral positions, for small losses $v_{g}$ can either be positive or negative, while for higher values it approaches zero, and finally all effects disappear and there is almost no anomalous propagation. On the other hand, at A, instead of a broad slow light regime there are only narrow resonances for certain absorptions and thickness. Moreover, it is important to note that for a sufficiently high absorption the superluminal character may persist even for thick samples. A similar behavior is found when light propagates in the GX direction.
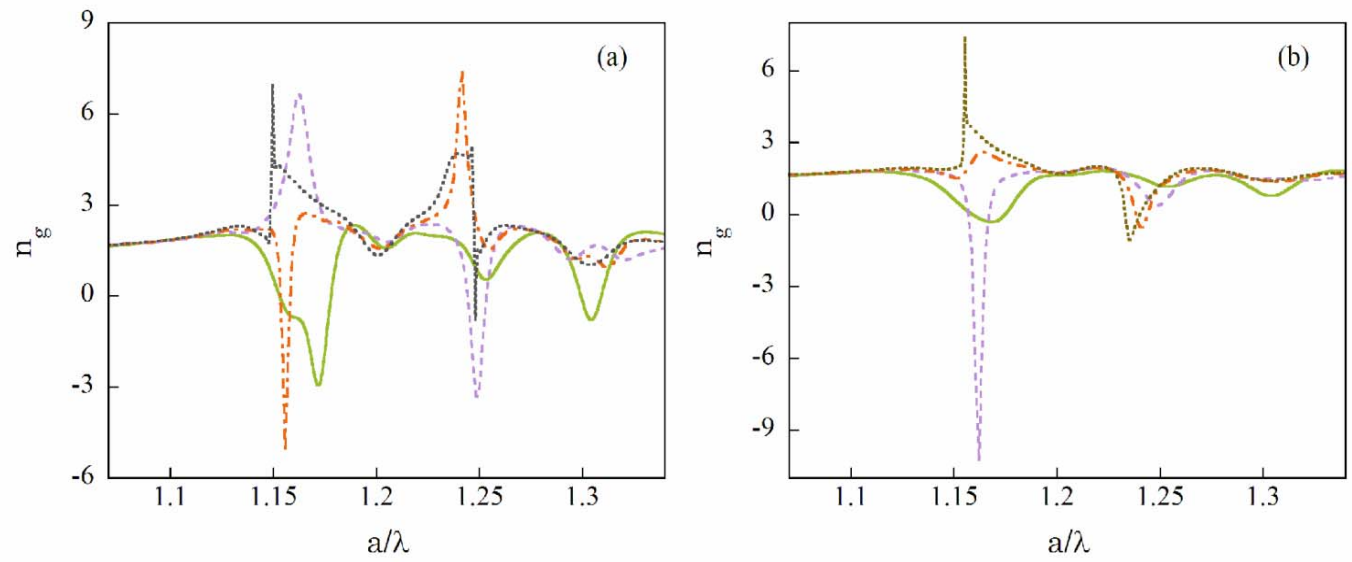

Figure 4. Group index in the $\Pi \mathrm{L}$ direction of a glass supported opal made of an fcc lattice of polystyrene spheres in air, for different extinctions: a) $\varepsilon_{s p h}=2.5+0.06 i$ (b) $\varepsilon_{s p h}=2.5+0.10 i$. The curves correspond to different opal thickness, in [111] planes: 6 (green solid curve), 10 (violet dashed curve), 20 (orange dashed-dotted curve), 200 (dotted black curve). All curves are plotted as a function of reduced frequency.

\section{CONCLUSIONS}

In summary, we have used vector KKR method with extinction to theoretically determine the dependence of $v_{g}$ on real aspects of thin opal-like structures, such as the finite-size effects or the role of extinction at the high energy range. We see that the group index, determined from the phase delay introduced to the forwardly transmitted field, evidences nonconventional light propagation at such energy range. In the $\Gamma L$ crystallographic direction we observe three frequency ranges where the finiteness of the structure gives rise to strong fluctuations of the $v_{g}$. We observe a complex optical behavior: $v_{g}$ becomes either superluminical, positive or negative or approaches zero, depending on the crystal length. We also determine that the difference in the phase ship delays, across the $v_{g}$ jumps, correspond to $2 \pi$. The above calculated group index reproduces very accurately previously reported experimental results for thin opal samples made of a reduced number of layers [5]. Moreover, the finitesize effects persist increasing the crystal thickness. We have also been able to describe the phase anomalies measured in the ГX crystallographic direction of thin artificial opals, reported in ref. [4]. Besides, we have shown that, at the high energy range, $v_{g}$ has also a strong dependence on imperfections. For simplicity reasons we have modeled the crystal disorder through an imaginary dielectric constant that accounts for extinction [15]. For a given crystal length, the presence of phase jumps in the transmitted electric field and superluminal light propagation regimes, depends on such crystal imperfections. The fact that for small extinctions, these effects persist even for a large number of planes indicates once again, that the finite character of the structure plays a crucial role even for relatively thick samples. Moreover, lattice disorder, losses and finite-size effects may represent a limiting factor to the predicted attainable minimum group velocities associated with the presence of low dispersion bands at that energy range. Increasing the crystal thickness smoothes the group index profiles but does not give rise to a broad fast light regime and still some thin fast light resonances persist. Therefore, we have showed that at the high-energy range of a finite periodic structure, real aspects as imperfections and finite-size effects can no longer be ignored to predict light propagation. Although great effort has always been made to avoid structural imperfections in largely ordered nanostructures, we find out that precisely disorder and finitesize effects lead to interesting new optical properties that could open new ways for the control of light. 


\section{REFERENCES}

[1] Y. A. Vlasov, M. O'Boyle, H.F. Hamann, S. J. McNab, Active control of slow light on a chip with photonic crystal waveguides, Nature 438, 65-69, 2005.

[2] D. Dahan, G. Eisenstein, Tunable all optical delay via slow and fast light propagation in a Raman assisted fiber optical parametric amplifier: a route to all optical buffering, Opt. Exp. 13,6234-6249, 2005.

[3] K. Sakoda, Optical properties of photonic crystals, Springer-Verlag, Berlin, 2005.

[4] J. F. Galisteo-López, M. Galli, A. Balestreri, L. C. Andreani, C. López, Optical response of artificial opals oriented along the ГX direction, Appl. Phys. Lett. 90,231112, 2007.

[5] J. F. Galisteo-López, M. Galli, A. Balestreri, M. Patrini, L.C. Andreani, C. López, Slow to superluminal light waves in thin 3D photonic crystals, Opt. Exp. 15, 15342, 2007.

[6] L. A. Dorado, R. A. Depine, G. Lozano, H. Míguez, Interplay between crystal-size and disorder in the highenergy optical response of photonic crystals slabs, Phys. Rev. B 76, 245103, 2007.

[7] M. Botey, J. Martorell, L. A. Dorado, R. A. Depine, G. Lozano, H. Míguez, Anomalous group velocity at the high energy range of a 3D photonic nanostructure, Opt. Exp. 18, 15682-15690, 2010.

[8] M. Botey, M. Maymó, A. Molinos-Gómez, L. A. Dorado, R. A. Depine, G. Lozano, A. Mihi, H. Míguez, J. Martorell, Second Harmonic Generation at the High Energy Range in a Nonlinear Opal Film, Opt. Exp. 17, 12210, 2009.

[9] M. Scharrer, A. Yamilov, X. Wu, H. Cao, R. P. H. Changb, Ultraviolet lasing in high-order bands of threedimensional ZnO photonic crystals, Appl. Phys. Lett. 88, 201103, 2006. H. Noh, M. Scharrer, M. A. Anderson, R. P. H. Chang, H. Cao, Photoluminescence modification by a high-order photonic band with abnormal dispersion in ZnO inverse opal, Phys. Rev. B 77,115136, 2008.

[10] X. Ma, J. Q Lu, R. S. Brock, K. M. Jacobs, P. Yang, X.-H. Hu, Determination of complex refractive index of polystyrene microspheres from 370 to 1610 nm, Phys. in Med. and Bio. 48,4165-4172, 2003.

[11] N. Stefanou, V. Karathanos, A. Modinos, Scattering of electromagnetic waves by periodic structures, J. Phys.: Condens. Matter 4, 7389-7400, 1992.

[12] N. Stefanou, V. Yannopapas, A. Modinos, Heterostructures of photonic crystals: Frequency bands and transmission coefficients, Phys. Commun. 113, 49-77, 1998.

[13] K. Ohtaka, Scattering theory of low-energy photon diffraction, J. Phys. C: Solid State 13 (1980) 667-680.

[14] A. Modinos, Scattering of electromagnetic waves by a plane of spheres-formalism, Physica A 141,575-588, 1987.

[15] L. A. Dorado and R. A. Depine, Modeling of disorder effects and optical extinction in three-dimensional photonic crystals, Phys. Rev. B 79, 045124, 2009.

[16] M. Botey, M. Maymó, J. Martorell, Band-structure determination for finite 3-D photonic crystals, Appl. Phys. B 81, 277-281, 2005. 